\title{
Maladaptive Cognitive Bias in the New Normal Period: An Analyses from a Behavioural Science Perspective in the Time of Covid-19
}

\author{
Zafira Rahmania Nur Shabrina ${ }^{1^{*}}$, Insan Rekso Adiwibowo², Nurul Aisya Beryllia ${ }^{3}$ \\ 1,3Faculty of Psychology, Universitas Gadjah Mada \\ ${ }^{2}$ Center for Policy and Health Management Faculty of Medicine, Nursing and Community \\ Health, Universitas Gadjah Mada
}

Submitted 23 October 2020 Accepted 30 November 2020 Published 22 December 2020

\begin{abstract}
The limited number of effective medical interventions to combat Covid-19 to date has resulted in government institutions focusing on preventive behaviours believed to minimize virus transmission. The Indonesian government has launched a 'new normal' campaign whereby outdoor activities are restricted by various health protocols established by health authorities, such as wearing a mask, habitual hand washing and social distancing. However, these protocols have not been implemented with a thorough understanding of human behaviour. The result is numerous violations of the protocol, which subsequently lead to the persistence of Covid-19 cases in Indonesia. Behavioural science as an approach can provide important insights regarding the systematic errors of thought that contribute to non-compliance with Covid-19 health signs. This article will elaborate on the different types of systematic errors, known as cognitive biases, that plays a role in Covid-19 protocol compliance and suggest the corresponding solutions deemed most effective to overcome these obstacles. Understanding of the dynamics paired with the application of behaviourally informed strategies will hence contribute to the attempt to flatten the Covid-19 curve.
\end{abstract}

Keywords: Covid-19; cognitive bias; new normal; protocol compliance

\section{Introduction}

The emergence of a novel coronavirus (SARS-CoV-2) disease in December 2019, officially referred to as Covid-19, has been announced as a global health crisis in March 2020 (World Health Organization, 2020). Between March to April 2020, numerous regions in Indonesia have implemented Large Scale Social Restrictions (Pembatasan Sosial Berskala Besar-

\footnotetext{
${ }^{1}$ Address for correspondence:
}

zrnshabrina@ugm.ac.id
PSBB) which obliges the closures of offices, schools, places of worship, and other public spaces. This policy has had a severe impact on the business sector, with the demands for goods and services plummeting along with declines in tourism and commodity prices.

As a result of the economic downturn and the continued upward trend of Covid19 cases in Indonesia, the PSBB policies have been relaxed, and numerous public activities resumed. To mitigate virus transmission amidst this relaxation, a set 
of health protocols were introduced. In general, the protocols outlined the actions that are necessary to prevent the transmission of Covid-19, namely using masks, regular hand washing, maintaining a one-meter distance from other people, and engaging in a hygienic lifestyle.

Relaxation of PSBB and introduction of such protocols can be interpreted as the shift of safety responsibility from the government (i.e., via partial lockdown) to individuals in the society (i.e., by abiding the protocol). However, to ensure that the protocols are effective in flattening the Covid-19 curve, the formation of new habits that adhere to health protocol needs to occur on a massive scale. Yet evidence shows that a vast majority of individuals are not ready or willing to engage in a behavioural change. For instance, attempts to promote the use of mask, has not been well accepted in numerous countries such as the United Kingdom, United States, Australia, and Finland (Advani, Yarrington, Smith, Anderson, \& Sexton, 2020; Gandhi \& Rutherford, 2020; Kai, Goldstein, Morgunov, Nangalia, \& Rotkirch, 2020; Smith, 2020).

Violations of health protocol cannot arguably be attributed to ignorance alone. Numerous behavioural theories have been used to explain the difficulties of encouraging compliance with health advice. Non-compliance can partially be attributed to cognitive bias which may lead to faulty reasoning of the Covid-19 Pandemic (Bottemanne, Morlaàs, Fossati, \& Schmidt, 2020; Halpern, Truog, \& Miller, 2020). Cognitive bias in the framework of behavioural science can be seen as a systematic error in the thinking process (Kahneman, 2003) and a systematic deviation from the norms or principles of rationality in a judgment (Haselton et al., 2015). This view argues that limited cognitive capacity and time constraints in decision making tend to make people base their decisions on intuition and heuristics (Tversky \& Kahneman, 1974; Thaler \& Sunstein, 2008; Thaler, 2016; Thaler, 2018). Therefore, cognitive bias occurs when people rely on their faulty intuition or when they fail to engage in elaborative cognitive processing in making a decision.

Even though it is seen as fault or error in the thinking process, cognitive biases have essential evolutionary functions. Cognitive bias allows humans to avoid harmful situations, choose non-poisonous food, and engage in social interaction (Haselton, Nettle \& Andrews, 2015). For instance, Arkes (1991) suggests that heuristics help minimize cognitive effort and avoid the use of strategies with costs that outweigh the benefits. Engaging in a complex thinking process for simple issues might require the development of new neural circuits in the brain that consumes a lot of energy for little benefit. A lot of decisions for survival purposes rely heavily on such intuitional heuristics because most of life or death situations require quick and spontaneous reaction although they may be far from optimal. However, in unfamiliar situations, doing tasks which require accurate judgment, or in a complex modern society, these tendencies may lead to fatal implications (Saposnik, Redelmeier, Ruff, \& Tobler, 2016).

During Covid-19 pandemic, cognitive biases can lead people to think and behave against scientific advice. Cognitive bias in the government level and policymaking may lead to the denial of science and 
epidemiological principles, subsequently resulting in many casualties. Cognitive bias at the community level can lead to difficulties in cutting the chain of transmission in dealing with the virus. This happens not necessarily due to lack of trust toward science; instead, it may be due to the embedded cognitive biases which often operates unconsciously and divert action against the best interest of epidemiological control.

The article will attempt to borrow insights from the field of behavioural science to delve in the cognitive biases that hold back individuals from adhering to Covid-19 health protocols. Specifically, this article will discuss compliance and non-compliance toward rules of using a mask, regular hand washing, and physical distancing. The recommendations stated in this article are not only relevant to the improvement of the Indonesian government's health protocols, but may also be applied to improve epidemic mitigation in school, workplaces, restaurants, and public facilities.

The Challenge of Cognitive Bias in Adopting New Habits

Cognitive biases emerge due to differences between individual's subjective perception and objective reality (Kahneman \& Tversky, 1996). Although in some cases, cognitive bias may be adaptive since it can simplify reality and lead to efficient decision making, it can also distort perception and lead to inaccurate judgment, illogical interpretations, as well as other irrational instances (Baron, 2008; Kahneman \& Tversky, 1972).

There are a number of cognitive biases which can prevent people from complying with the Covid-19 health protocols, such as wearing a mask, habitual hand washing, and maintaining a physical distance. Among the cognitive biases that will be discussed are optimism bias, status-quo bias, loss aversion/probability weighting, and present bias.

\section{Optimism Bias}

Optimism bias refers to a cognitive fallacy in human judgment whereby a person believes that the probability of them being negatively impacted by an event is small. Conversely, they believe that their probability of receiving a positive outcome is larger compared to other people (Weinstein, 1980; Weinstein 1987; Sharot, 2011). In simple terms, optimism bias can be defined as the difference between expectations of better outcomes as compared to reality (Sharot, 2011). The inability to objectively evaluate situation may lead to engagement in risky behaviours, such as risky sex and smoking (e.g., van der Pligt, Otten, Richard, \& van der Velde 1993; Weinstein, Marcus, \& Moser, 2005), and also non-compliance to the Covid-19 health protocols. Individuals with optimism bias may refuse to wear a mask, even though other people who have daily or close interaction with them choose to wear one. This is because optimism bias makes a person believe that their probability of contracting the virus is lower compared to other people, hence the judgement that no decision (i.e., wearing masks, avoid crowds, or wash hands) needs to be taken.

\section{Default Options Bias}

The default options bias refers to individual preferences in maintaining status 
quo and resisting change despite leading to positive outcomes (Samuelson \& Zeckhauser, 1988; Loewenstein Brennan, \& Volpp, 2007; Thaler \& Sunstein, 2008). Thaler and Sunstein (2008) attribute the lack of attention towards one's options in the novel situation as one of the causes of this bias.

This bias can explain why some individuals do not wear masks as a way to prevent infection of Covid-19, as their lives prior to the pandemic did not require the use of masks. Although wearing a mask is advised and is a preferable option during the pandemic, this change of behaviour is difficult since it has not become a default option. In addition, such novelty may increase the probability of a person forgetting to engage in the behaviour. Moreover, individuals may interpret that the costs gained from changing the status quo are too large to incur compared to the benefits (Soofi, Najafi, \& Kamari-Matin, 2020). Using a mask for a sustained period can be uncomfortable for some people and may cause breathing difficulty and skin irritation. This cost of mask-wearing is perceived as larger because the inconvenience happens immediately and consistently compared to the delayed uncertain benefit of wearing masks to avoid Covid-19 infection.

\section{Loss Aversion and Probability Weighting}

Loss aversion and probability weighting are not concepts derived from the prospect theory of Kahneman and Tversky (1979). Loss aversion refers to one's tendency to be more sensitive towards a loss than a gain. In managing a loss, people tend to avoid a certain loss, although the loss is small compared to a large but uncertain loss. As an illustration, if a person with loss aversion has to confront the following options: (a) losing Rp100.000 with a 100\% certainty or (b) losing Rp200.000 with a $50 \%$ certainty. According to prospect theory, the person will tend to prefer loss of Rp200.000 with a 50\% certainty.

Probability weighting refers to one's subjective evaluation towards risk that follows a non-linear utility function (Kahneman \& Tversky, 1979). It means that people tend to overvalue the smallest probability and undervalue the highest probability as long as the likelihood of an event is not absolute ( 0 or 1$)$. Various campaigns on Covid-19 put emphasize on how doing social activities bring a high risk of Covid-19. These campaigns, despite bringing awareness on how infectious Covid-19 is, could bring hyperbolic undervaluing of the risk for some people with intertemporal discounting tendency or gambling tendency. In the face of absolute cost of inconvenience in wearing a mask (probability equal to 1), an uncertain cost of contracting Covid-19 is perceived as significantly lower than the inconvenience even though the probability value is near to 1 .

In adhering to health protocols, individuals with loss aversion will choose the costlier option with lower probability; that is, not following the advised protocols and relying on sheer luck of not being infected. Compliance with the protocols is costlier because it contains both immediate direct and indirect costs. In straightforward terms, a person has to purchase numerous items such as masks, hand hygiene products, hair tie, and additional clothing. The indirect costs may include discomfort when complying with the 
protocols, such as feeling hot and hard to breathe due to wearing mask or difficulties in finding a place to wash hands. Both costs, for most people, should be paid to follow the advised protocols and the price might be too painful for some so that a little risk of getting infected can be ignored.

\section{Present Bias}

The present bias refers to the tendency of preferring immediate rewards to delayed rewards as well as delayed costs to immediate costs (O'Donoghue \& Rabin, 1999; Loewenstein, John, \& Volpp, 2012). This bias explains why people have difficulties engaging in behaviours that promise maximum (yet delayed) benefit in the future, such as doing taking preventive actions for future health problems (van der Pol, Hennessy, \& Manns, 2017).

In the context of Covid-19, the present bias may explain why people do not obey the protocols of physical distancing and avoid crowds in public places. Violating Covid-19 protocols, such as going to leisure centres and engaging in conversations with a group of friends in close physical proximity, is perceived as an immediate reward that is much preferred compared to staying at home to avoid the risk of infection which is perceived to bring delayed rewards of not contracting the virus. Additionally, because the Covid19 virus itself is microscopic, the impacts of maintaining distance with other people to prevent virus transmission cannot be directly captured by the human senses. This intangibility reduces the perceived magnitude and immediacy of avoiding the disease as a reward. According to Weber and Chapman (2005), humans are motivated to conduct actions that have measurable and real outcomes that can be captured by the senses as opposed to those that are intangible and undetected by the senses.

Why does Cognitive Bias Lead to Irrational Decisions?

Cognitive bias is a form of cognitive performance and in some contexts may be considered as irrational. Nevertheless, from an evolutionary perspective, these functions may be adaptive to a person's survival. One popular perspective to explain the emergence of bias is error management theory (Haselton \& Buss, 2000). The main principle from this theory is that there is a general cognitive mechanism that can result in two types of errors: false positives (taking action when it is undesirable), and false negatives (failure to take action when it is desirable). According to the error management theory, most errors in human judgment reflect a mechanism designed to make errors that are minor but often occur, compared to those that are fatal but rare (Haselton \& Buss, 2000; Haselton et al., 2015). A more detailed illustration of error management which leads to adaptive functions in the context of Covid-19 can be seen in Table 1.

In the context of the Covid-19 Pandemic as an extreme event, where information is scarce and uncertain, this ancestral mechanism operates and overlooks the risks of Covid-19 as a form of cost due to the following reasons: (1) lack of time to internalize the cost of Covid-19 within bounded rationality; (2) presence of obstacles in internalizing such information due to beliefs, values and 
motivated reasoning; and (3) feedback from cognitive bias which prevents change from current existing cognitive schemes.

This may lead to maladaptive cognitive bias in the Covid-19 situation, which occurs as a function of time. As an example, in early January when the SARS$\mathrm{CoV}-2$ was in its initial transmission phase in China, many assumed that the virus would only spread in China and saw only a small probability that the virus would reach other countries, thereby exemplifying optimism bias. A person's evaluation of SARS-CoV-2 changes constantly with the increase of information to the point that people become aware of the threat the virus presents. The illustration shown in Table 1. delineates the dynamics of cognitive bias which will emerge as a natural response when confronting a situation with limited information and high uncertainty (or in Bayesian terms, biased prior). The development or absence of an initial response with other information depends on many factors.

\section{How Problematic are These Cognitive Biases?}

Optimism bias, status-quo/default option, loss aversion and probability weighting, as well as present bias, are just several examples of bias that may prevent people from adhering to Covid-19 health protocols. However, other cognitive errors and factors may also be at play in preventing one from taking preventive action. One factor that may contribute to non-compliance in wearing a mask and washing hands is forgetfulness. Meanwhile, physical distancing, desire to participate in social gatherings, and inability to control other people's behaviour also present challenges in engaging in preventive actions.

Table 1.

Cognitive Bias According to Error Management Theory in the Context of Covid-19

\begin{tabular}{llll}
\hline \multicolumn{1}{c}{ Bias } & $\begin{array}{c}\text { Comparison of the costs of } \\
\text { False Positives (FP)-False } \\
\text { Negatives (FN) }\end{array}$ & $\begin{array}{l}\text { Adaptive } \\
\text { function }\end{array}$ & $\begin{array}{c}\text { Maladaptive in Covid-19 } \\
\text { situation }\end{array}$ \\
\hline Optimism Bias & $\begin{array}{l}\text { Cost when not acting (FN) is } \\
\text { larger compared to the cost of } \\
\text { acting when incapable of doing } \\
\text { so (FP) (Nettle, 2004). }\end{array}$ & $\begin{array}{l}\text { Ambition, } \\
\text { persistence, } \\
\text { invention. }\end{array}$ & $\begin{array}{l}\text { Undermine risk, threat, } \\
\text { overconfidence. }\end{array}$ \\
\hline Status-quo Bias & $\begin{array}{l}\text { Cost of creating new neural } \\
\text { networks in the brain to resist } \\
\text { the new complex situation is } \\
\text { larger compared to accepting the } \\
\text { status-quo (Fleming, Thomas, \& } \\
\text { Dolan, 2010) }\end{array}$ & $\begin{array}{l}\text { Stability, } \\
\text { security, } \\
\text { energy } \\
\text { efficiency. }\end{array}$ & $\begin{array}{l}\text { Resist changing } \\
\text { behaviours which adhere } \\
\text { to safety protocols to } \\
\text { manage the pandemic. }\end{array}$ \\
\hline
\end{tabular}




\begin{tabular}{|c|c|c|c|}
\hline Loss aversion & $\begin{array}{l}\text { In general, the marginal loss has a } \\
\text { large effect on survival compared } \\
\text { to receiving a marginal gain } \\
\text { (McDermott, Fowler, \& Smirnov, } \\
\text { 2008) }\end{array}$ & Survival & $\begin{array}{l}\text { Unwilling to lose current } \\
\text { liberties: going to a } \\
\text { restaurant, breathing } \\
\text { without using a masker, } \\
\text { gathering with friends. }\end{array}$ \\
\hline
\end{tabular}

Overcoming Cognitive Limitations with Behavioural Science

In addition to helping explain the various problems that may arise due to cognitive bias which subsequently that may prevent individuals from observing health protocols to contain the Covid-19 virus, behavioural science also provides the solution to manage these cognitive limitations. The solution which is offered in this article falls within the nudge strategies. Nudge is a concept in the field of behavioural economics that refers to efforts of persuading individuals to act rationally and making desirable choices without eliminating other choices (Thaler \& Sunstein, 2008). Thaler and Sunstein (2008) often refer to nudge as choice architecture which is used to create a conducive environment for individuals to make easy choices. Past research has shown that the effectiveness of nudge in the context of health, for example in reducing cigarette and alcohol consumption as well as increasing fruit and vegetable consumption and exercise (Marteau, Ogilvie, Roland, Suhrcke, \& Kelly, 2001). Some articles published about the pandemic also argued that nudge is a solution to overcome non-adherence to protocols and other medical advice related to Covid-19 (Soofi et al., 2020; Hume, John, Sanders, \& Stockdale, 2020).

As mentioned above, by using nudge strategies, cognitive bias can be taken advantage to persuade a person's behaviour. Therefore, while the previous sections focused on cognitive biases as a hindrance of health protocol compliance, this section will discuss the use of cognitive bias to direct adherence of health protocols during the Covid-19 pandemic. Examples of recommendations that are given in this section are just a sample of the many possible nudge techniques. It shall also be stated that its effectiveness depends on the context of its application. Nevertheless, the examples below can illustrate the use of nudge in managing the Covid-19 pandemic in Indonesia.

\section{Using Framing Effects}

Framing effects refers to a bias in decision making when equivalent objective information is presented differently, namely either in positive or negative terms, which leads to change in choice preferences (Kahneman \& Tversky, 1979; Tversky \& Kahneman, 1981). Furthermore, Tversky and Kahneman (1981) found that individuals tend to avoid risk (i.e., risk aversion) when related with situations that are beneficial and engage in risk-seeking when it relates with situations that associates with a loss. For messages that promote preventive behaviours, individuals tend to like information that is presented in a way that shows a gain of performing a behaviour compared to framing the behaviour in terms of a loss (Gallagher \& Updegraff, 2012). 
The effects of this framing can be used in increasing compliance with Covid-19 health protocols. For example, Jordan, Yoeli, and Rand (2020) found that messages which focus on collective interests (e.g., “don't spread it") were more effective in increasing Covid-19 preventive behaviours compared to messages that focus on the ego (e.g. "don't get it.") In line with Jordan et al. (2020), Lunn et al. (2020) found that messages which focused in one as the source of a virus-spreading are more effective in increasing compliance compared to messages that explicitly ask people to maintain a 2-meter distance with other people. Both research results support Sasaki, Kurokawa, and Ohtake (2020), who found that altruistic messages are more effective compared to egoistic ones.

\section{Using Social Norms}

Human behaviour is largely affected by social norms, namely, perception of what other people are doing or is perceived as the appropriate action. Conformity towards social norms is conducted because the need to affiliate and a need to be accepted (Cialdini \& Goldstein, 2004). However, there is often a misperception or estimation error related to what other people are doing. Therefore, one of the strategies that can be taken creating normbased messages.

As far as the author is aware, there has been no research during Covid-19 that tests the use of norms-based messages despite its effectiveness in other contexts. For example, Goldstein, Cialdini and Griskevicius (2008) found that normsbased messages are more effective compared to a standard message in increasing reuse of towels in the hotel ("75\% of people staying in the hotel reuse their towels" [re: do not ask for a second towel in the second day] compared to a message "HELP SAVE THE ENVIRONMENT. Show your respect for nature and help save the environment by reusing your towels." If conducted in the context of Covid-19, the message " $80 \%$ of people comply to mask-wearing protocol when leaving the house" (the percentage is not factual and is only used for illustration) would be more effective compared to a message "Save lives by using a mask when leaving your home."

Furthermore, research from Centola (2011) found that perceptions related to norms were stronger when demonstrated or delivered by someone with the same identity. Therefore, messages to encourage protocol compliance can be delivered by religious leaders as well as other respected figures. Sending the message and developing an effective monitoring system need to be applied through social networks such as friends, relatives and colleagues (Bavel et al., 2020).

Making a Compensation System Based on the Principles of Present Bias

The present bias causes an individual to engage in actions to receive immediate rewards over actions to delay immediate gratification in favour of obtaining maximum future benefits ( $\mathrm{O}^{\prime}$ Donoghue \& Rabin, 1999). This same mechanism can also be used as a foundation to increase compliance to Covid-19 health protocols. One of the solutions is by reducing the cost that must be incurred or increasing the rewards that are gained when complying with the protocol (Soofi et al., 2020). As an 
example, the government or supplier of goods and services can give immediate rewards for people who choose to maintain physical distance and stay at home (e.g., giving discounts for internet access and free delivery for people who chose to purchase goods online) and simplify procedures related to public service for the people (e.g., online applications for processing of National Identity Cards or Driving License).

\section{Conducting Risk Communication by} Increasing Information on Loss

In the prospect theory popularized by Kahneman and Tversky (1979), people tend to avoid certain loss and prefer uncertain loss despite the larger implications. In the context of Covid-19, a certain loss can mean a cost, discomfort, and complex protocols which are undesirable compared to "only" being infected by the virus. To overcome the problem, the effect of framing and loss aversion needs to be combined. As an illustration, compared to delivering a message "be aware of Covid-19 by complying with health protocols," risk communication can be done by giving two messages simultaneously. The first message delivers a loss and the injunction to comply with the protocol. However, the message is communicated in a way that allows people to identify with the message, such as "wearing a mask makes it difficult to breathe", "don't have any money to purchase hand sanitizer?", and "the protocols are too much of a hassle". The second message delivers the large loss using a third-person narrative, such as "Covid-19 leads to permanent damage of the lungs", "Covid-19 can infect your family and the people you love", and "Covid-19 patients will be buried alone, is this what you want?". By framing the message in such a way, people will compare the loss that is experienced from the first message and judge that it is not as large as the impact of Covid-19 infection delivered in the second message. Subsequently, the cognitive bias that emerges from the consideration of opportunities would neutralize the more sensitive message that leads to resistance to loss. Of course, the excess use of this technique needs to consider the side effects that emerge due to apathy which have been evident in smoking warnings included in advertisements.

\section{Conclusion}

While awaiting medical breakthrough that cures Covid-19, there is an urgent need to encourage compliance of health protocols in order to slow down the transmission of the virus in Indonesia as well as the rest of the world. Although efforts have been made to promote health behaviours at scale, the results suboptimal with yet any signs of the curve flattening. Conversely, violations of health protocols continue to be observed in Indonesia. This article elaborates the reason behind the noncompliance in three behaviours deemed most important in reducing virus transmission: handwashing and hygiene, socialdistancing etiquette and mask use.

This article argues that noncompliance with protocols is attributed to cognitive biases, namely systematic errors in thinking processes. Cognitive bias in the context of non-compliance towards Covid19 occurs due to limits of cognitive 
capacity to make sense of the complex, uncertain and novel situations. These cognitive limitations subsequently lead to the use of intuition in decision making. Five cognitive biases were discussed, namely optimism bias, status-quo, loss aversion and opportunity weighting, and present bias.

Despite its adverse consequences, past behavioural research in numerous contexts suggests that cognitive biases can be overcome using a range of strategies. Three strategies are recommended to increase compliance with Covid-19 health protocols, including message-framing that supports risk communication, use of social norms, and compensation systems based on principles of present bias. However, it must be noted that the advised strategies require empirical support for its effectiveness in the context of compliance toward Covid-19 protocols, despite evidence pointing its success in other contexts.

This article emphasizes the importance of behaviourally informed interventions and mass communication. Evidence and examples provided in this paper are expected to inform public health mass communication as well as aid the development of behavioural interventions in Indonesia - ultimately reducing transmission of the COVID-19 virus as the society and economy begin to reopen. Suggestions provided in this paper are not only relevant for the improvement of health protocols designed by the Indonesian government, but may also be applied in the workplace, entertainment establishments, and other organizations.

\section{References}

Advani, S. D., Yarrington, M. E., Smith, B. A., Anderson, D. J., \& Sexton, D. J. (2020). Are we forgetting the "universal" in universal masking? Current challenges and future solutions. Infection Control and Hospital Epidemiology, 1-2. doi: $\quad$ 10.1017/ice. 2020.333

Arkes, H. R. (1991). Costs and benefits of judgment errors: Implications for debiasing. Psychological Bulletin, 110(3), 486-498. doi: 10.1037/0033$\underline{2909.110 .3 .486}$

Baron, J. (2000). Thinking and deciding. Cambridge: Cambridge University Press.

Bavel, J. J. V., Baicker, K., Boggio, P. S. et al. (2020). Using social and behavioural science to support Covid-19 pandemic response. Nat Hum Behav, 4, 460-471. doi: 10.1038/s41562-020-0884$\underline{\mathrm{Z}}$

Bickel, W. K., Moody, L., \& Higgins, S. T. (2016). Some current dimensions of the behavioural economics of healthrelated behaviour change. Preventive medicine, 92, 16-23. doi: 10.1016/j.ypmed.2016.06.002

Bottemanne, H., Morlaàs, O., Fossati, P., \& Schmidt, L. (2020). Does the coronavirus epidemic take advantage of human optimism bias? Frontiers in Psychology, 11(August), 1-5. doi: 10.3389/fpsyg.2020.02001

Burns, Z., Chiu, A., \& Wu, G. (2010). Overweighting of small probabilities. In J. J. Cochran (Ed.), Wiley encyclopedia of operations research and management science (pp.1-8). New York: Wiley. doi: 10.1002/9780470400531.eorms0634

Centola, D. (2011) An experimental study of homophily in the adoption of health 
behaviour. Science, 334(6060), 12691272. doi: $10.1126 /$ science. 1207055

Cialdini, R. B. \& Goldstein, N. J. (2004). Social influence: compliance and conformity. Annu Rev Psychol. 55(44), 591-621.

doi: $\underline{10.1146 / \text { annurev.psych.55.090902.1420 }}$ $\underline{15}$

Fleming, S. M., Thomas, C. L., \& Dolan, R. J. (2010). Overcoming status quo bias in the human brain. Proceedings of the National Academy of Sciences of the United States of America, 107(13), 60056009. doi: $10.1073 /$ pnas.0910380107

Gallagher, K. M., \& Updegraff, J. A. (2012). Health message framing effects on attitudes, intentions, and behaviour: $\mathrm{A}$ meta-analytic review. Annals of Behavioural Medicine, 43(1), 101-116. doi: 10.1007/s12160-011-9308-7

gandhi, m., \& rutherford, g. w. (2020). facial masking for covid-19 potential for "variolation" as we Await a Vaccine. New England Journal of Medicine, 383(18), e101. doi: 10.1056/NEJMp2026913

Goldstein, N. J., Cialdini, R. B., \& Griskevicius, V. (2008). A room with a viewpoint: Using social norms to motivate environmental conservation in hotels. Journal of Consumer Research 35, 472-482. ㅇi: $\underline{10.1086 / 586910}$

Halpern, S. D., Truog, R. D., \& Miller, F. G. (2020). Cognitive bias and public health policy during the COVID-19 pandemic. JAMA, 324(4), 337. doi: 10.1001/jama.2020.11623

Haselton, M. G., \& Buss, D. M. (2000). Error management theory: A new perspective on biases in cross-sex mind reading. Journal of Personality and Social Psychology, 78(1), 81-91. doi: $\underline{10.1037 / 0022-3514.78 .1 .81}$
Haselton, M. G., Nettle, D., \& Andrews, P. W. (2005). The handbook of evolutionary psychology. The Evolution of Cognitive Bias, 724-746.

Haselton, M. G., Nettle, D., \& Andrews, P. W. (2015). The evolution of cognitive bias. The Handbook of Evolutionary Psychology, 724-746.

Hume, S., John, P., Sanders, M., \& Stockdale, E. (2020). Nudge in the time of Coronavirus: The persistence of behavioural messages during crisis. SSRN Electronic Journal. doi: 10.2139/ssrn

Jordan, J., Yoeli, E., \& Rand, D. (2020). Don't get it or don't spread it? Comparing self-interested versus prosocially framed Covid-19 prevention messaging. Working paper, https://psyarxiv.com/yuq7

Kahneman, D. (2003). Maps of bounded rationality: Psychology for behavioural economics. American Economic Review, 93, 1449-1475. doi: $\underline{10.1257 / 000282803322655392}$

Kahneman, D., \& Tversky, A. (1979). Prospect theory: An analysis of decision under risk. Econometrica, 47(2), 263-291. doi: 10.2307/1914185

Kahneman, D., \& Tversky, A. (1996). On the reality of cognitive illusions. Psychological Review, 103, 582-591. doi: 10.1037/0033-295x.103.3.582

Kai, D., Goldstein, G. P., Morgunov, A., Nangalia, V., \& Rotkirch, A. (2020). Universal masking is urgent in the COVID-19 Pandemic: SEIR and agent based models, empirical validation, policy recommendations. ArXiv.

Loewenstein, G., Brennan, T., \& Volpp, K. G. (2007). Asymmetric paternalism

to improve health behaviours. Journal of the American Medical Association, 298(20), 
2415-2417.

\subsection{1/jama.298.202415}

Loewenstein, G., John, L. K., \& Volpp, K.G. (2012). Using decision errors to help people help themselves. In E. Shafir (Ed.), The behavioural foundations of public policy (pp.361-379). Princeton, NJ: Princeton University Press.

Lunn, P. D., Timmons, S., Barjaková, M., Belton, C. A., Julienne, H., \& Lavin, C. (2020). Motivating social distancing during the Covid-19 Pandemic: An online experiment. ESRI Working Paper, 658.

Marteau, T. M., Ogilvie, D., Roland, M., Suhrcke, M., \&; Kelly, M. P. (2011). Judging nudging: Can nudging improve population health? BMJ, 342(3), 228. doi: 10.1136/bmj.d228

McDermott, R., Fowler, J. H., \& Smirnov, O. (2008). On the evolutionary origin of prospect theory preferences. Journal of Politics, 70(2), 335-350. doi: $10.1017 /$ S0022381608080341

Nettle, D. (2004). Adaptive illusions: optimism, control, and human rationality. In Emotion, Evolution, and Rationality (pp. 193-208). doi: 10.1093/acprof:oso/9780198528975.003. $\underline{0010}$

O'Donoghue, T., \& Rabin, M. (1999). Doing it now or later. American Economic Review, 89(1), 103-124. doi: 10.1257/aer.89.1.103

Samuelson, W., \& Zeckhauser, R. (1988). Status quo bias in decision making. Journal of Risk and Uncertainty, 1(1), 759. doi: 10.1007/BF00055564

Saposnik, G., Redelmeier, D., Ruff, C. C., \& Tobler, P. N. (2016). Cognitive biases associated with medical decisions: a systematic review. BMC Medical Informatics and Decision Making, 16(1), 1-14. doi: 10.1186/s12911-016-0377-1
Sasaki, S., Kurokawa, H., \& Ohtake, F. (2020). Short-term responses to nudgebased messages for preventing the spread of Covid-19 infection: Intention, behaviour, and life satisfaction. Discussion Papers in Economics and Business No. 20-11, Osaka University, Graduate School of Economics. Retrieved from https://econpapers.repec.org/paper/os kwpaper/2011.htm

Sharot, T. (2011). The optimism bias. Current Biology, 21(23), R941-R945. doi: 10.1016/j.cub.2011.10.030

Smith, M. (2020). International COVID-19 tracker update: 2 September. Retrieved November 16, 2020, from YouGov Covid-19 Tracker website:

https://today.yougov.com/topics/healt $\mathrm{h} /$ articles-

reports/2020/09/02/international-covid19-tracker-update-2-september

Soofi, M., Najafi, F., \& Karami-Matin, B. (2020). Using insights from behavioural economics to mitigate the spread of Covid-19. Applied Health Economics and Health Policy, 18, 345350. doi: $10.1007 / \mathrm{s} 40258-020-00595-4$

Thaler, R. H. (2016). Behavioural economics: Past, present, and future. American Economic Review, 106(7), 1577-600. doi: 10.1257/aer.106.7.1577

Thaler, R. H. (2018). From cashews to nudges: The evolution of behavioural economics. American Economic Review, 108(6), 1265-1287. doi: $\underline{10.1257 / a e r .108 .6 .1265}$

Thaler, R. H., \& Sunstein, C. R. (2008). Nudge: Improving decisions about health, wealth and happiness. New Haven, CT: Yale University Press.

Tversky, A., \& Kahneman, D. (1974). Judgment under uncertainty: Heuristics and biases. Science, 185(4157), 
1124-1131.

10.1126/science.185.4157.1124

Tversky, A., \& Kahneman, D. (1981). The framing of decisions and the psychology of choice. Science, 211(4481), 453-458. $\quad$ doi: 10.1126/science. 7455683

van der Pligt, J., Otten, W., Richard, R., \& van der Velde, F. (1993). Perceived risk of AIDS: unrealistic optimism and selfprotective action. In J. B. Pryor, \& G. D. Reeder (Eds.). The social psychology of HIV-infection (pp.39-58). Hillsdale, NJ: Lawrence Erlbaum.

van der Pol, M., Hennessy, D., \& Manns, B. (2017). The role of time and risk preferences in adherence to physician advice on health behaviour change. The European Journal of Health Economics, 18(3), 373-386. doi: 10.1007/s10198-016-0800-7

Weber, B. J., \& Chapman, G. B. (2005). Playing for peanuts: Why is risk seeking more common for low-stakes gambles? Organizational Behaviour and Human Decision Processes, 97(1), 31-46. doi: 10.1016/j.obhdp.2005.03.001
Weinstein, N. D. (1980). Unrelistic optimism about future life events. Journal of Personality and Social Psychology, 39(5), 806-820. doi: 10.1037/00223514.39.5.806

Weinstein, N. D. (1987). Unrealistic optimism about susceptibility to health problems: Conclusions from a community-wide sample. Journal of Behavioural Medicine, 10(5), 481-500. doi: $10.1007 / \mathrm{BF} 00846146$

Weinstein, N. D., Marcus, S. E., \& Moser, R. P. (2005). Smokers' unrealistic optimism about their risk. Tobacco Control, 14(1), 55-59. doi: 10.1136/tc. 2004.008375

World Health Organization (2020). WHO announces Covid-19 outbreak a pandemic. Retrieved September 23, 2020, from:

https://www.euro.who.int/en/healthtopics/healthemergencies/coronavirus-Covid19/news/news/2020/3/who-announcesCovid-19-outbreak-a-pandemic 